\title{
Beta-adrenergic Modulation of Cognitive Flexibility during Stress
}

\author{
Jessica K. Alexander, Ashleigh Hillier, Ryan M. Smith, \\ Madalina E. Tivarus, and David Q. Beversdorf
}

\begin{abstract}
Stress-induced activation of the locus ceruleus-norepinephrine (LC-NE) system produces significant cognitive and behavioral effects, including enhanced arousal and attention. Improvements in discrimination task performance and memory have been attributed to this stress response. In contrast, for other cognitive functions that require cognitive flexibility, increased activity of the LC-NE system may produce deleterious effects. The aim of the present study was to determine the effect of pharmacological modulation of the LC-NE system on stress-induced impairments in cognitive flexibility performance in healthy individuals. Cognitive performance, plus psychological and physiological parameters for 16 adults without any history of anxiety disorders, was assessed during four test sessions: stress and no-stress, with each condition tested after administration of propranolol and placebo. The Trier Social Stress Test, a public-speaking and mental arithmetic stressor,
\end{abstract}

\section{INTRODUCTION}

The ability to think in a flexible manner has been proposed as a critical component of creativity and insight, and some studies have suggested that it is susceptible to the deleterious effects of stress (Martindale \& Greenough, 1973). Cognitive flexibility is the capacity to inhibit a dominant response when it represents a nonoptimal or inappropriate solution to a problem, and to enable access to more remote alternatives. For our purposes, cognitive flexibility will refer to flexibility of access to the lexical-semantic and associative network in a verbal problem-solving task (Kelley, Yeager, Pepper, \& Beversdorf, 2005; Beversdorf, White, Chever, Hughes, \& Bornstein, 2002; Beversdorf, Hughes, Steinberg, Lewis, \& Heilman, 1999), in contrast to other forms of cognitive flexibility such as set-shifting. The likelihood of a dominant, nonflexible response increases during high arousal states (Easterbrook, 1959), and stressors heighten arousal (Koob, 1991; Johnson \& Anderson, 1990; Koob, Cole, Swerdlow, Le Moal, \& Britton, 1990). Innovative problem solving is therefore thought to be negatively affected

The Ohio State University was presented to participants for the stress sessions, whereas a similar, but nonstressful, control task (reading, counting) was utilized for the no-stress sessions. Tests of cognitive flexibility included lexical-semantic and associative problem-solving tasks (anagrams, Compound Remote Associates Test). Visuospatial memory and motor processing speed tests served as control tasks. Results indicate that (1) stress impaired performance on cognitive flexibility tasks, but not control tasks; (2) compared to placebo, cognitive flexibility improved during stress with propranolol. Therefore, psychological stress, such as public speaking, negatively impacts performance on tasks requiring cognitive flexibility in normal individuals, and this effect is reversed by beta-adrenergic antagonism. This may provide support for the hypothesis that stress-related impairments in cognitive flexibility are related to the noradrenergic system.

by stress, as several studies have suggested (Heilman, Nadeau, \& Beversdorf, 2003; Ashby, Isen, \& Turken, 1999). A remaining uncertainty is the primary mechanism underlying stress-induced impairments of cognitive flexibility.

Many studies examining cognitive flexibility have examined the effect of the serotonergic (Evers et al., 2005; Clarke, Dailey, Crofts, Robbins, \& Roberts, 2004) and dopaminergic (Floresco, Magyar, Ghods-Sharifi, Vexelman, \& Tse, 2006) systems. However, stressors impact the locus ceruleus-noradrenergic system (LC-NE) to stimulate the release of norepinephrine (NE) (Berridge \& Waterhouse, 2003; Segal \& Bloom, 1976). In general, behavioral studies suggest that increased noradrenergic activity enhances processing of salient stimuli concomitant with suppression of irrelevant stimuli; in effect, NE acts to narrow attentional focus (Aston-Jones, Rajkowski, \& Cohen, 1999; Coull, Buchel, Friston, \& Frith, 1999; Coull, 1998). Furthermore, increased distractibility can result from decreased NE activity (Coull, Jones, Egan, Frith, \& Maze, 2004; Smith \& Nutt, 1996; Bunsey \& Strupp, 1995; Arnsten \& Contant, 1992). These may occur as a result of the effect of $\mathrm{NE}$ on the signal-to-noise ratio within cortical neurons (Hasselmo, Linster, Patil, Ma, \& 
Cekic, 1997). Although the enhanced "signal" aspect of the ratio would be related to superior performance on tasks of attention, the behavioral role of "noise" is less understood. Based on a computational model, however, it has been suggested that "noise" is a representation of intrinsic associative activity, not simply an absence of coherent input to cortical neurons (Hasselmo et al., 1997). Related to this model, we propose that the increased intrinsic associative activity of "noise" occurring with decreased NE relates to improved performance on cognitive flexibility tasks involving flexibility of access to the lexical-semantic and associative network (Beversdorf et al., 1999, 2002). Our previous research has suggested a modulatory role of the noradrenergic system on this type of cognitive flexibility performance (Beversdorf et al., 1999, 2002), which may explain the deleterious effects of arousal and stress. Furthermore, because performance after administration of propranolol (a central and peripheral beta-adrenergic receptor blocker) was significantly better than after nadolol (a peripheral-only beta-adrenergic receptor blocker), this effect appears to be centrally mediated (Beversdorf et al., 2002). However, neither drug had a significant effect as compared to placebo in an unstressed condition.

Recent research has suggested that phasic LC activity, driven by outcome of task-related decisions believed to be computed in the orbito-frontal and anterior cingulate cortices, facilitates task-relevant processes in contrast to distractors to optimize task performance (Aston-Jones \& Cohen, 2005). Increased tonic LC activity, particularly when associated with decreased phasic LC activity, promotes disengagement from current behaviors while facilitating exploration of other behaviors (Aston-Jones \& Cohen, 2005). As would be expected by this tonic LC effect, increased alpha-1 adrenergic activity has been shown to facilitate attentional set-shifting (Lapiz \& Morilak, 2006). However, flexibility of access to the lexical-semantic and associative network may not be affected in the same manner. Stress has been shown to impair other aspects of prefrontal cortical function (Arnsten, 2000), a region throughout which NE stimulation from the LC is received (Lewis \& Morrison, 1989), and which is, in general, important for performance on a wide variety of aspects of cognitive flexibility (Stemme, Deco, Busch, \& Schneider, 2005; Dalley, Cardinal, \& Robbins, 2004). Furthermore, some of these aspects of prefrontal cortical function are impaired by stressinduced beta-1 adrenergic activity (Ramos et al., 2005) and alpha-1 adrenergic activity (Birnbaum, Gobeske, Auerbach, Taylor, \& Arnsten, 1999).

Therefore, our purpose was to utilize a well-established model of psychosocial stress, the Trier Social Stress Test (TSST) (Kirschbaum, Pirke, \& Hellhammer, 1993), involving public speaking and mental arithmetic, in order to examine the effect of psychosocial stress on cognitive flexibility and the effect of beta-adrenergic antagonists on this response. In order to examine the proposed effects of alterations of intrinsic associative activity, our cognitive flexibility tasks required a broad search of the lexical-semantic and associative network to find the solution. We expected stress to impair performance on these cognitive flexibility tasks, an effect which would be attenuated by propranolol, a nonspecific beta-adrenergic antagonist. Performance on visuospatial memory tasks, previously demonstrated not to be affected by conditions with increased NE tone (Kelley et al., 2005), and motor processing speed were also measured within the significant time constraints of the pharmacological and stress model in order to control for general effects on cognitive performance and processing speed.

\section{METHODS}

\section{Participants}

Participants were 16 healthy adults ( 8 men, 8 women) with a mean age of 23.81 years $(S D=4.69)$, who were recruited through flyers posted on the Ohio State University campus. Exclusion criteria included a known history of learning disability, dyslexia, phobia to math, or giving speeches or any other anxiety disorder, and risk factors for adverse reaction to beta-adrenergic blockers (known history of depression, diabetes, thyroid disease, pulmonary disease, bradyarrhythmias, narrow angle glaucoma, congestive heart failure, previous adverse reactions to beta-blockers, and asthma). In addition, nonnative English speakers; smokers; those already taking beta-blockers; and those unable to comply with a 12-hour caffeine, alcohol, and intense exercise abstention prior to all sessions were excluded. Procedures were performed in accordance with the Biomedical Sciences Institutional Review Board of the Ohio State University. A written informed consent form was obtained from all participants.

\section{Experimental Design}

The participants reported to the General Clinical Research Center (GCRC) for five sessions, with at least 3 days between each of the four experimental sessions. The first session was designated for obtaining baseline physiological measurements. Following a 15-min adaptation period, heart rate (HR) was recorded continuously for $6 \mathrm{~min}$ while the participants rested in a private room with minimal disturbance. Blood pressure (BP) was measured every 5 min during this period.

Over the course of the four experimental sessions, the participants experienced each of the following conditions in a double-blind, counterbalanced manner: (1) Placebo + Control, (2) Placebo + Stress, (3) Propranolol $40 \mathrm{mg}+$ Control, and (4) Propranolol $40 \mathrm{mg}+$ Stress. For each experimental visit, as with the baseline visit, following a 15-min adaptation period, HR was recorded 
continuously for $6 \mathrm{~min}$, and BP data were collected twice, at minutes 1 and 6. Propranolol $40 \mathrm{mg}$ or placebo was then administered, followed by a period of $45 \mathrm{~min}$ during which participants rested without physiological measurements. Then, physiological monitoring resumed with continuous HR monitoring and BP measurements every $5 \mathrm{~min}$ for the remainder of the session. The instructions for the modified TSST Stress or Control tasks were relayed to the participant (Kirschbaum et al., 1993). An assessment of anticipatory stress (Stress Appraisal Measure [SAM]) (Peacock \& Wong, 1990) was obtained after participants were informed of the task. As with our previous work using propranolol (Beversdorf et al., 2002), cognitive testing began $60 \mathrm{~min}$ following drug administration. Participants continued to be monitored for $3 \mathrm{~min}$ following the end of the cognitive testing. After completing the final experimental session, participants were debriefed regarding the purpose and procedures associated with the stress protocol.

\section{Stress and Control Tasks}

A modified TSST (Kirschbaum et al., 1993) was utilized to elicit stress and to determine its effects on cognitive performance. Participants were asked to deliver a 5-min speech supporting their candidacy for employment in a law office or for admission into graduate business school. The speech task was followed by a 5-min mental arithmetic task. These were performed in front of panelists wearing white lab coats, taking notes, and instructed not to provide signs of positive reinforcement. A video camera and audio recorder were present during the stress condition, and participants were informed that their performance would be subsequently judged by behavioral analysts. For the control tasks, participants were asked to read aloud a designated passage and count alone (with no panelists or video camera) in a room, each for $5 \mathrm{~min}$. Administration of the first cognitive task began when participants were interrupted 1 min after the start of the speech or passage reading task. Following conclusion of the cognitive test, the TSST verbal component (speech task or reading aloud) was restarted. At 1- or 2-min timed intervals, the TSST was again interrupted and the subsequent cognitive tests were performed. Participants were informed to focus on the cognitive task at hand, rather than be concerned about keeping track of their place within the verbal or mathematical task, and that the experimenters would remind them of where they had stopped in their speech or arithmetic task.

\section{Cognitive Tests}

\section{Cognitive Flexibility Tests}

In order to assess cognitive flexibility, one of four versions of the Compound Remote Associates test (CRA) (Bowden
\& Jung-Beeman, 2003) was administered at each experimental session. This test required participants to generate a word associated with three presented words by formation of a compound word (e.g., the solution for "child," "wash," and "scan" is "brain"). The participant was allowed 7 sec per triad for 15 triads per session. Solutions were scored for accuracy. The CRA test is reported to measure insight-based problem solving and is similar to the Remote Associates Test (Mednick, 1962), previously utilized as a test of creativity.

As a further measure of cognitive flexibility, one of four versions of the anagram task was also administered at each experimental session. This required unscrambling three 5-letter words, six 6-letter words, and six 7-letter words, each word within $30 \mathrm{sec}$. Three separate presentations (one presentation per interruption) of five anagram problems (each containing one 5-letter word and two of both 6- and 7-letter words) were presented within each session. As with our previous work (Beversdorf et al., 1999, 2002), natural log (ln) of latency to solve the anagram was recorded and summed for each session to derive latency scores. Anagram problem solving has been utilized previously as a method of assessing creativity (Shaw \& Conway, 1990; Kumar \& Kumari, 1988; Gavurin, 1975). Previous evidence suggests that performance on this task is modulated by the noradrenergic system (Silver, Hughes, Bornstein, \& Beversdorf, 2004; Beversdorf et al., 1999, 2002).

\section{Visuospatial Memory and Motor Speed}

In order to assess functions other than cognitive flexibility within the time constraints of this experiment, tests of visuospatial construction and memory and motor processing speed were also administered. One of four versions of the Rey-Osterrieth Complex Figure Test (CFT) (Corwin \& Bylsma, 1993; Rey, 1941) was administered at each experimental session. This assesses visuospatial constructional ability and visual memory (Rey, 1941). Participants were asked to accurately copy a complex figure and $30 \mathrm{~min}$ later reproduce it from free recall. This was scored for accuracy on a 36-point scale. Within the time limitations of the drug and stress conditions, this served as a control for other cognitive domains that do not principally involve cognitive flexibility. The grooved pegboard (GP) (Lafayette Instruments, Lafayette, IN) test was also administered at each experimental session in order to measure processing speed as well as visual-motor coordination and manual dexterity. Participants were asked to insert grooved pegs into randomly positioned slots on the board as quickly as possible. Performance is determined by speed of completion. This served as a control for processing speed, as latency was used for the anagram task.

The order of test versions was presented in a counterbalanced manner across conditions. 


\section{Physiological Measures}

For the physiological data, in each of the sessions, five disposable spot electrodes were placed in a five-lead configuration for continuous HR measurement, and a BP cuff was placed on the participants' nondominant arm (GE DASH 4000 telemetry device, Milwaukee, WI). Each session was divided into four time epochs for physiological data collection: baseline (following a 15-min adaptation period; $6 \mathrm{~min}$ in duration), anticipatory ( $45 \mathrm{~min}$ postdrug administration and prior to start of condition, during speech/reading preparation; $10 \mathrm{~min}$ ), intraexperimental (during stress or control; 20-30 min), and recovery (following completion of experiment; $3 \mathrm{~min}$ ). As described above, systolic blood pressure (SBP) and diastolic blood pressure (DBP) were collected every 5 min during each epoch, and HR was assessed continuously throughout the sessions and was analyzed and presented for each epoch (baseline, anticipatory, and recovery; intraexperimental was analyzed and presented in 3-min epochs due to the comparatively longer collection period).

\section{Self-reported Stress Measure}

Participants rated the anticipated stressfulness of each session during the anticipatory phase of the session (after receiving instructions and preparing for the task, but prior to the start of the speech or reading task). Participants rated their perception of the task on a 5-point Likert scale on the SAM (Peacock \& Wong, 1990).

\section{Statistical Analysis}

Physiological, psychological, and cognitive data were analyzed using analysis of variance (ANOVA), comparing across time points (baseline vs. anticipatory, intraexperimental, and recovery) for the physiological measures, and for all measures between drug conditions (placebo vs. propranolol), and between the two experimental sessions (control vs. stress). Post hoc comparisons were analyzed using one-way ANOVA or paired-sample $t$ tests.

\section{RESULTS}

\section{Physiological Measures}

To confirm that the stressors elicited the expected physiological response of sympathetic activation, cardiovascular data were compared between the stress session and the control session. As expected, a significant condition (stress vs. control) by time point (baseline BP vs. intraexperimental BP) interaction using a two-way ANOVA was observed for SBP $[F(1,15)=12.736, p=$ $.003]$ and DBP $[F(1,15)=9.697, p=.007$; see Figure 1$]$. No such effect was observed with the comparison of baseline BP versus anticipatory BP or baseline BP versus recovery BP. Post hoc analyses showed that the stress condition significantly increased SBP and DBP during the experiment as compared to baseline $[t(15)=-4.03$, $p=.001 ; t(15)=-4.67, p<.001$, respectively), whereas the control condition had no effect on BP. Between
Figure 1. Mean BP between experimental sessions. Each time point represents the average of data collected every $5 \mathrm{~min}$ throughout the experiment. Significant differences $(p<.05)$ at indicated time points are denoted by the following symbols: (*) for Placebo Stress vs. Placebo Control groups; $(\dagger)$ for Placebo Stress vs. Propranolol Stress; ( $\ddagger$ ) for Placebo Stress vs. Propranolol Control; (\#) for Placebo Control vs. Propranolol Control; (+) for Placebo Control vs. Propranolol Stress; and $(x)$ for Propranolol Stress vs. Propranolol Control. Error bars represent mean standard error.

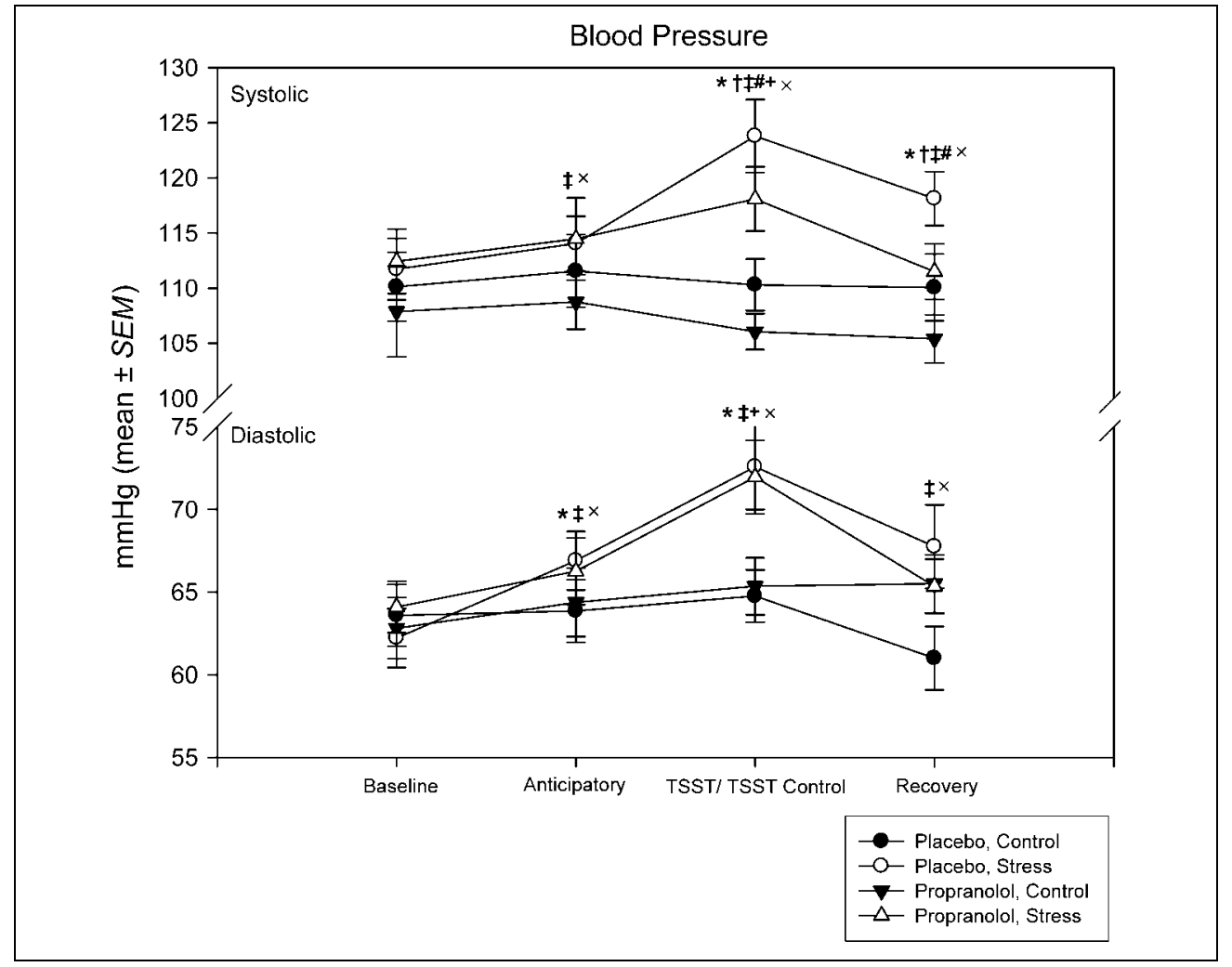


conditions, BP was not significantly increased during the baseline or anticipatory phases of the stress session compared to the control condition; however, SBP was elevated during the recovery phase of the stress condition compared to the control condition $[F(1,15)=$ 4.656, $p=.048$ ]. The average HR (beats per minute) was used to calculate a mean $\mathrm{HR}$ for the baseline, anticipatory, and recovery periods (Figure 2). The intraexperimental period was divided into a series of 3-min interval means for HR. An initial effect of condition was observed during the intraexperimental period of the stress session $[F(1,15)=6.885, p=.019]$, in which HR increased compared to baseline measurements during the first $3 \mathrm{~min}$ of the task. HR did not increase significantly from baseline during any period of the control condition, nor for the anticipatory or recovery period of the stress condition.

As expected, propranolol exerted sympatholytic effects on measures of physiological reactivity. Analysis for drug effects focused on the baseline versus intraexperimental time point comparison, as this was the only comparison affected by stress as described above. Threeway ANOVA revealed significant main effects of drug [placebo vs. propranolol; $F(2,15)=10.133, p=.006$ ], condition [stress vs. control; $F(2,15)=36.939, p<$ .001 ] , and time point [baseline SBP vs. intraexperimental SBP; $F(2,15)=5.123, p=.039$ ] for SBP (Figure 1). There were no significant interactions among drug and condition. Post hoc analyses indicated that, compared to placebo, propranolol reduced SBP during the intraexperimental phase of both the control $[F(1,15)=$ $5.597, p=.032]$ and the stress conditions $[F(1,15)=$ 8.353, $p=.011]$. Consistent with previous studies, DBP was unaffected by propranolol (Maheu, Joober, \&
Lupien, 2005; Maheu, Joober, Beaulieu, \& Lupien, 2004). For each time point, two-way ANOVA for condition (stress vs. control) and drug (placebo vs. propranolol) indicated that propranolol significantly decreased HR during all phases of the experiment, and in both conditions, in anticipation of the experiment $[F(1,15)=$ $10.621, p=.005]$, during the experiment $[F(1,15)=$ $17.060, p=.001]$, and during the recovery epoch $[F(1,15)=21.230, p<.001]$ (except baseline, before the drug was administered) (Figure 2). These results indicate that propranolol exerted a significant effect on measures of sympathetic nervous system reactivity.

\section{Self-reported Anticipatory Stress}

Participants rated expected perceived stress of each session during the anticipatory phase of the session. For anticipatory stress (SAM), a main effect of stress with no drug effect or interaction effect revealed that participants rated the stress condition to be more stressful as compared to the control condition $[F(1,15)=$ $38.627, p<.0001$ ], regardless of propranolol or placebo treatment.

\section{Cognitive Performance}

As shown in Table 1, Figure 3 (CRA), and Figure 4 (anagrams), compared to the control condition, the stress condition was associated with significantly impaired performance on measures of cognitive flexibility (CRA and anagrams), but not memory or motor coordination tasks (CFT and GP) during the placebo sessions. Oneway ANOVAs showed that, compared to performance in
Figure 2. Mean HR between experimental sessions. Each time point represents the mean of all participants' HR for which 3 min of data was averaged per participant. Significant differences $(p<$ $.05)$ at indicated time points are denoted by the following symbols: (*) for Placebo Stress vs. Placebo Control groups; $(\dagger)$ for Placebo Stress vs. Propranolol Stress; $(\ddagger)$ for Placebo Stress vs. Propranolol Control; (\#) for Placebo Control vs. Propranolol Control; and $(+)$ for Placebo Control vs. Propranolol Stress. Error bars represent mean standard error.

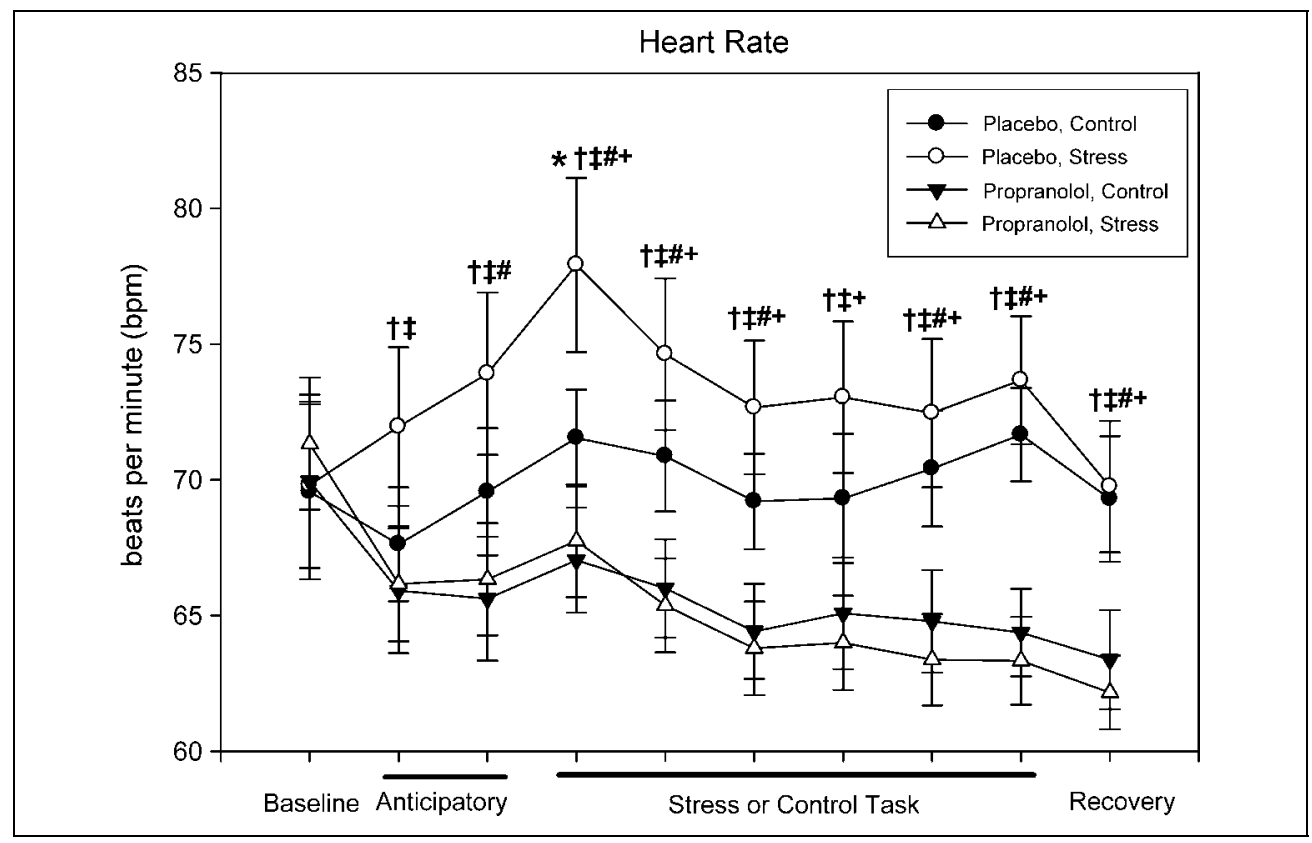


Table 1. Performance on Cognitive Tasks with and without Stress on Placebo and Propranolol (Mean $\pm S E$ )

\begin{tabular}{|c|c|c|c|c|}
\hline \multirow[b]{2}{*}{ Measure } & \multicolumn{4}{|c|}{ Testing Condition } \\
\hline & Placebo Control & Placebo Stress & Propranolol Stress & Propranolol Control \\
\hline \multirow[t]{2}{*}{ CRA (number correct) } & $7.75 \pm 0.74$ & $6.56 \pm 0.67$ & $8.19 \pm 0.70$ & $7.94 \pm 0.60$ \\
\hline & \multicolumn{2}{|c|}{$p=.04 *$} & $p=.05^{* *}$ & \\
\hline \multirow[t]{2}{*}{ Anagrams (ln sec to complete) } & $29.14 \pm 1.83$ & $31.68 \pm 1.81$ & $28.65 \pm 1.89$ & $27.59 \pm 2.10$ \\
\hline & \multicolumn{2}{|c|}{$p=.03^{*}$} & $p=.05 * *$ & \\
\hline \multirow[t]{2}{*}{ Rey CFT (scored for accuracy) } & $25.22 \pm 1.67$ & $23.88 \pm 1.70$ & $22.03 \pm 1.80$ & $25.44 \pm 1.67$ \\
\hline & \multicolumn{2}{|c|}{$p=.30$} & $p=.88$ & \\
\hline \multirow[t]{2}{*}{ Grooved pegboard (sec to complete) } & $65.57 \pm 3.27$ & $66.53 \pm 3.95$ & $65.57 \pm 4.02$ & $67.14 \pm 2.92$ \\
\hline & \multicolumn{2}{|c|}{$p=.67$} & .49 & \\
\hline
\end{tabular}

CRA = Compound Remotes Associates test; CFT = Complex Figure Test.

One-way ANOVAs shown for placebo control versus placebo stress and placebo stress versus propranolol stress.

* Placebo-stress significantly different from placebo-control.

***Propranolol-stress trend-to-significantly different from placebo-stress.

the control condition, in the stress condition, the number of correct responses declined for the CRA test $[F(1,15)=5.094, p=.04]$, and anagram solution latency scores increased $[F(1,15)=5.576, p=.03]$ without drug. Compared to the control condition, no effect of stress was observed for measures of visuospatial memory $[$ CFT; $F(1,15)=1.162, p=.30]$ or the motor speed task $[\mathrm{GP} ; F(1,15)=0.192, p=.67]$ without drug.

Propranolol reversed stress-induced impairment of cognitive flexibility, improving performance levels to within the range achieved during the control conditions.
Specifically, propranolol enhanced the ability to solve CRA problems during stress as compared to placebo during stress. A significant Condition (control vs. stress) $\times$ Drug (placebo vs. propranolol) interaction using ANOVA was observed for the CRA test $[F(1,15)=5.064, p=.04]$. Post hoc analyses revealed a trend-to-significant increase in correct solutions with propranolol during stress compared to placebo $[F(1,15)=4.409, p=.05]$, as well as the increased number of correct solutions with placebo during the control condition compared to the stress condition as described above. A two-way
Figure 3. Mean $( \pm S E M)$ number correct responses on the Compound Remote Associates test for each condition. Compared to the control condition, stress impaired performance. Performance during the stress condition demonstrated a trend-to-significant improvement after propranolol as compared to placebo. An asterisk indicates significance at the level $p \leq .05$.

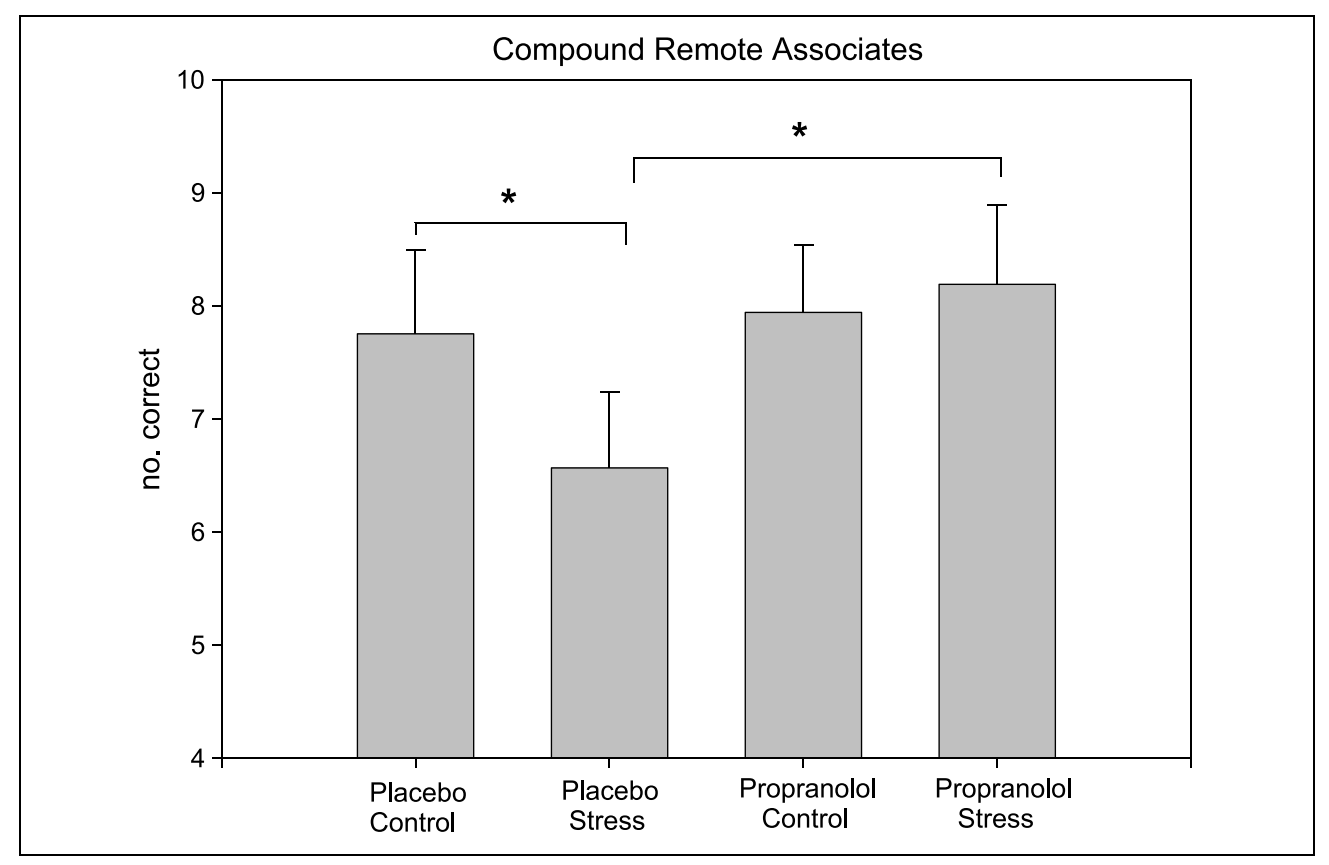


Figure 4. Mean $( \pm S E M)$ time to complete anagrams for each condition. Stress impaired performance compared to the control condition. Performance during the stress condition demonstrated a trend-to-significant improvement after propranolol as compared to placebo. An asterisk indicates significance at the level $p \leq .05$.

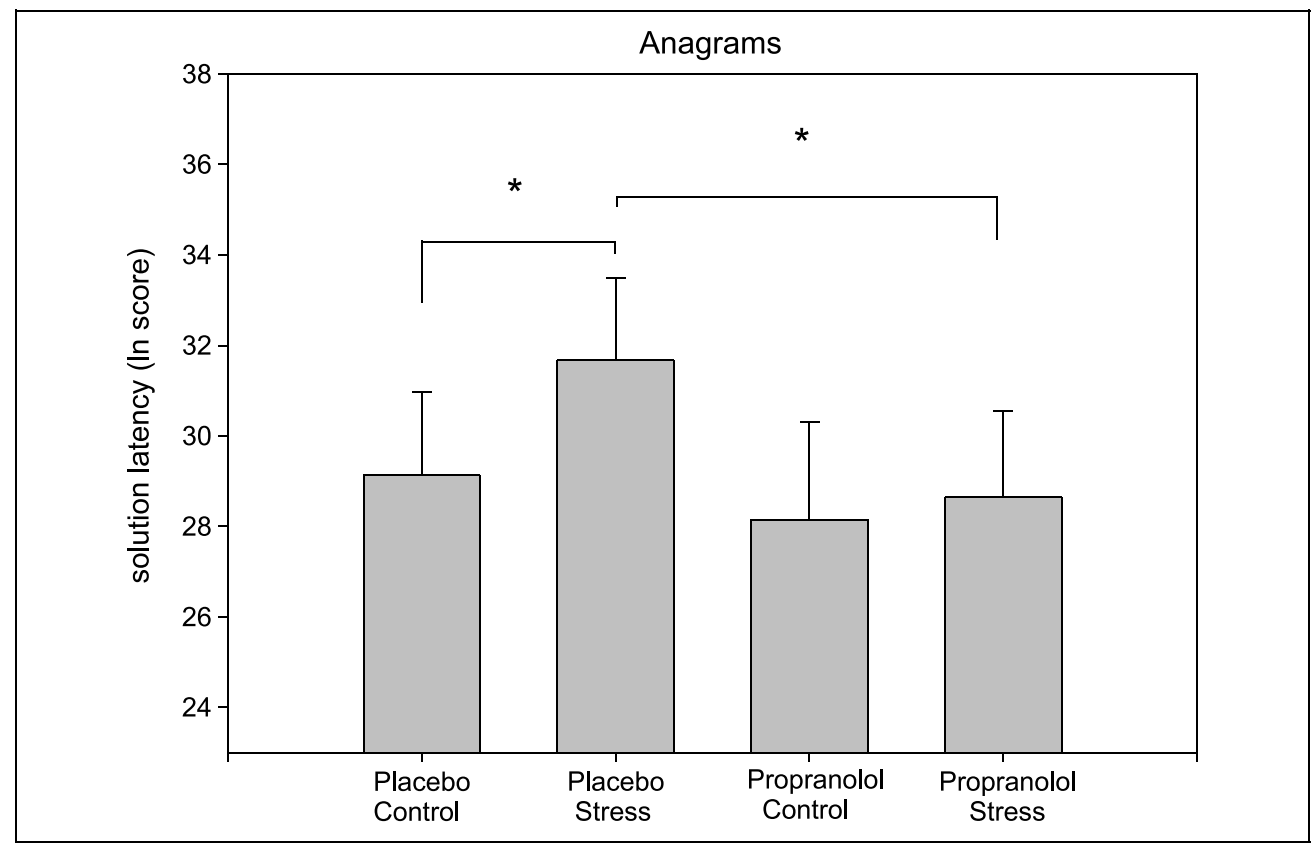

ANOVA examining condition (control vs. stress) and drug (placebo vs. propranolol) also revealed a significant Condition $\times$ Drug interaction $[F(1,15)=10.811, p=$ $.005]$ for anagram performance. Post hoc tests showed that latency scores for solving anagrams had a trend-tosignificant decrease with propranolol during stress compared to placebo $[F(1,15)=4.505, p=.05]$, as well as the decreased solution latency with placebo during the control condition compared to the stress condition as described above. During stress, neither visuospatial memory [CFT; $F(1,15)=1.634, p=.221$ ] nor motor speed $[\mathrm{GP} ; F(1,15)=0.236, p=.634]$ was affected by propranolol. Compared to placebo, no effect of propranolol was observed for cognitive performance on any task during the control condition.

\section{DISCUSSION}

In the present study, we observed an impairment of cognitive flexibility performance during an experimental stressor (see Figures 3 and 4). Compared to the control condition, participants showed significant impairments on tests of cognitive flexibility when exposed to a psychosocial stressor (TSST Stress condition). Furthermore, propranolol reversed the impairment resulting from the psychosocial stressor. During stress, individuals performed anagrams more quickly and also produced more correct responses on the CRA task after taking propranolol compared to placebo. Therefore, our results may suggest that the observed decline in flexibility is attributable to stress-induced increases in beta-adrenergic activity, as has been previously demonstrated in other aspects of executive function, where beta-1 adrenergic blockade improved working memory impairments resulting from stress-related increases in beta-1 activity (Ramos et al., 2005). Therefore, propranolol, as a nonspecific beta-adrenergic antagonist, would also block this cognitively detrimental stress pathway. Lack of an effect on visuospatial memory and motor speed suggests some specificity to cognitive flexibility; however, we cannot exclude a cognitive effect of other stress hormones. Administration of propranolol may affect complex interactions between the beta-adrenergic system and corticosteroid system, where activation of the latter has been shown to affect a number of aspects of cognition including many aspects attributed to frontal lobe function (Elzinga \& Roelofs, 2005; Maheu et al., 2004, 2005; de Quervain, Roozendaal, Nitsch, McGaugh, \& Hock, 2000; Lyons, Lopez, Yang, \& Schatzberg, 2000; Skosnik, Chatterton, Swisher, \& Park, 2000; Young, Sahakian, Robbins, \& Cowen, 1999), perhaps indirectly by blocking adrenergic activity. The effects of corticosteroids can also include anatomical changes in the prefrontal cortex (Cerqueira et al., 2005). Stress-induced catecholaminergic release does not occur after lesions of the amygdala (Goldstein, Rasmusson, Bunney, \& Roth, 1996), thus implicating amygdalar projections to the LC in the stress response. However, propranolol has also been shown to block corticosterone-induced impairment of working memory (Roozendaal, McReynolds, \& McGaugh, 2004). Therefore, future studies will need to monitor cortisol levels as well as physiological measures of stress and degree of NE blockade, including drug levels over time, in order to disentangle these effects.

Previous research has demonstrated impaired performance on tests requiring cognitive flexibility during conditions of experimentally induced arousal (Martindale \& 
Greenough, 1973). Further, Reich and Zautra (2002) have suggested that high stress results in simplified cognitive processing, such that the ability to simultaneously process information, discriminate among choices, and make judgments is impaired during stress. Cognitive rigidity is also associated with anxiety (Dey, 1978; Tomasini, 1973; Harleston, Smith, \& Arey, 1965), where drugs impacting the NE system demonstrate beneficial effects. For example, adolescents with known test anxiety benefited from propranolol with improved scores on the Scholastic Aptitude Test (SAT) (Faigel, 1991). However, this effect has not previously been investigated in individuals without known anxiety-induced impairments. Antianxiety agents that do not act on the NE system do not appear to have this effect on cognitive flexibility (Silver et al., 2004).

Stressors and other salient stimuli activate the LC-NE system (Berridge \& Waterhouse, 2003; Aston-Jones, Rajkowski, Kubiak, Valentino, \& Shipley, 1996; Segal \& Bloom, 1976). Behaviorally, this has the effect of increasing arousal (Aston-Jones et al., 1999; Koob, 1991; Johnson \& Anderson, 1990; Koob et al., 1990). High arousal states are associated with an inability to attend to competing cues, with the result of a narrowing of attention (Coull et al., 1999; Coull, 1998). Physiologically, activation of the LC-NE system increases noradrenergic neuronal firing rates, an effect which may inhibit processes of some forms of cognitive flexibility (Hasselmo et al., 1997). NE may modulate cognitive functioning through changes in the signal-to-noise ratio of cortical neuron firing properties (Nieuwenhuis, AstonJones, \& Cohen, 2005). However, the relationship between our findings and tonic and phasic activity of the LC is not yet clear. One would expect an increase in tonic LC firing with stress and a decrease of the influence of increased LC firing with propranolol, which would be expected to have the opposite effects on behavioral set-shifting (Aston-Jones \& Cohen, 2005; Nieuwenhuis et al., 2005) than what is observed in our cognitive flexibility task. Evidence supports increased adrenergic (alpha-1) activity exerting a facilitatory effect on set-shifting (Lapiz \& Morilak, 2006), an effect which is reversed by alpha-1 but not beta-adrenergic antagonists. Thus, in contrast to this alpha-1 effect, by reducing the influence of NE released by stress via the amygdala-LC stress pathway on the presumed prefrontal cortexmediated neuronal network search through betaadrenergic receptors (possibly beta-1 receptors; Ramos et al., 2005), propranolol may be facilitating conditions optimal for cognitive flexibility for the types of tasks used in our study. It is also possible that the constrained nature of selection from a limited number of options that typifies behavioral flexibility elicited by a set-shifting task ("constrained cognitive flexibility") benefits from increased tonic LC firing (Aston-Jones \& Cohen, 2005), whereas the broad unconstrained network search required in our tasks is optimized by relatively decreased tonic LC activity in order to decrease "signal" and increase the influence of the "noise" of intrinsic associational activity (Hasselmo et al., 1997) in prefrontal cortical neurons to access a more remote solution ("unconstrained cognitive flexibility").

Here we show that psychosocial stress in healthy individuals without any history of stress-induced dysfunction impairs performance on tasks that require flexible thinking, and that beta-adrenoceptor blockade reverses this performance decrement during stress. Visuospatial memory and motor processing speed were unaffected by stress and, also, by propranolol. The observed specificity of stress and propranolol to affect only cognitive flexibility suggests a role of the noradrenergic system in modulating the neural circuitry that may play a role in underlying such modalities as creativity and insight. That visuospatial memory and motor speed were not altered by stress or propranolol implies resistance to the effects of psychosocial stress and elevated $\mathrm{NE}$, perhaps as functions that are regionally positioned to receive less noradrenergic innervation or functions that are not regulated by beta-receptor activation. Thus, our observations suggest that beta-adrenergic blockade during stress permits increased access to more remote solutions, suggesting that elevated norepinephrine results in impaired access to solutions in problem solving during stress. Previous studies also have demonstrated a positive effect of noradrenergic suppression on problem solving and cognitive flexibility, specifically, in testanxious individuals (Faigel, 1991), and impairment in cognitive flexibility in patients believed to have upregulated NE activity due to cocaine withdrawal (Kelley et al., 2005). The current results were produced in a healthy study population without any known history of drug abuse or anxiety. Propranolol was therefore acting on a background of presumably "typical" noradrenergic responses to psychosocial stress, and not on neuronal circuitry associated with highly anxious or highly stressreactive individuals. In similar behavioral contexts, autonomic responses to stressors vary between individuals. Specifically, there is substantial variance in noradrenergic control of peripheral stress responses (Cacioppo et al., 1995; Berntson et al., 1994). However, noradrenergic control of cognitive flexibility appears in our study to have a somewhat consistent effect across a group of individuals. However, the significant time constraints of the pharmacological and stress model and the resulting limited number of cognitive tasks examined in this experiment limit the interpretation of these findings. The visuospatial memory task was selected because previous work suggested that it is not affected by noradrenergic tone (Kelley et al., 2005). Further studies will need to reexamine this issue with a variety of other verbal tasks, not dependent upon cognitive flexibility. As increased alpha-1 activity has been shown to facilitate attentional set-shifting (Lapiz \& Morilak, 2006), other types of cognitive flexibility tasks including set-shifting 
should be examined in this setting. Furthermore, drugs decreasing adrenergic activity have often demonstrated no effect under control conditions (Beversdorf et al., 1999, 2002), but reverse the cognitive effects of stress as well as other pharmacological challenges in other settings (Ramos et al., 2005; Roozendaal et al., 2004; Murphy, Arnsten, Jentsch, \& Roth, 1996). Further studies will then be needed to examine the effects of task difficulty in general, increases of which could decrease performance to suboptimal levels, allowing the potential cognitively enhancing effects of adrenergic antagonists to be detected. These factors (visual vs. verbal task selection and task difficulty) may also contribute to the lack of an effect of stress on memory in this study. Further work will also be necessary to examine the effects on processing speed beyond what was observed on the motor task in this experiment. Furthermore, the pharmacologic specificity of this effect must be examined, utilizing a range of alpha- and beta-specific NE agents and other anxiolytic agents as well as corticosteroidrelated agents. Better understanding of the anatomical pathways underlying this process is also needed. As a result of a similar pattern of stress and adrenergic effects found in working memory (Ramos et al., 2005; Birnbaum et al., 1999), one might speculate that the dorsolateral prefrontal cortex, which receives NE fibers from the LC (Lewis \& Morrison, 1989), may be a target of the proposed NE modulatory effects, resulting in effects on neural network searches regulated by engagement of the dorsolateral prefrontal cortex.

\section{Acknowledgments}

This study was supported by the General Clinical Research Center at The Ohio State University, grant M01-RR00034 from the National Center of Research Resources of the NIH, and by NIDA (DA15734) (Beversdorf) and NINDS (NS043222) (Beversdorf). Preliminary results of this research were presented in abstract form at Society for Neuroscience, 2006. We thank Heather Campbell, Ananth Narayanan, Adam Marcitceh, and Dr. Joseph Kitzmiller, for their help on the stressor panels. We would also like to thank Dr. Janice Kiecolt-Glaser for her advice on the stressor paradigm.

Reprint requests should be sent to David Q. Beversdorf, Department of Neurology, The Ohio State University, Means Hall 469, 1654 Upham Drive, Columbus, OH 43210 USA, or via e-mail: david.beversdorf@osumc.edu.

\section{REFERENCES}

Ashby, F. G., Isen, A. M., \& Turken, A. U. (1999). A neuropsychological theory of positive affect and its influence on cognition. Psychological Review, 106, 529-550.

Arnsten, A. F. T. (2000). Stress impairs prefrontal cortical function in rats and monkeys: Role of dopamine D1 and norepinephrine $\alpha-1$ receptor mechanisms. Progress in Brain Research, 126, 183-192.

Arnsten, A. F. T., \& Contant, T. A. (1992). Alpha-2 adrenergic agonists decrease distractibility in aged monkeys performing the delayed response task. Psychopharmacology, 108, 159-169.

Aston-Jones, G., \& Cohen, J. D. (2005). An integrative theory of locus coeruleus-norepinephrine function: Adaptive gain and optimal performance. Annual Review of Neuroscience, 28, 403-450.

Aston-Jones, G., Rajkowski, J., \& Cohen, J. (1999). Role of locus coeruleus in attention and behavioral flexibility. Biological Psychiatry, 46, 1309-1320.

Aston-Jones, G., Rajkowski, J., Kubiak, P., Valentino, R. J., \& Shipley, M. T. (1996). Role of the locus coeruleus in emotional activation. Progress in Brain Research, 107, 379-402.

Berntson, G. G., Cacioppo, J. T., Binkley, P. F., Uchino, B. N., Quigley, K. S., \& Fieldstone, A. (1994). Autonomic cardiac control. III. Psychological stress and cardiac response in autonomic space as revealed by pharmacological blockades. Psychophysiology, 31, 599-608.

Berridge, C. W., \& Waterhouse, B. D. (2003). The locus coeruleus-noradrenergic system: Modulation of behavioral state and state-dependent cognitive processes. Brain Research: Brain Research Reviews, 42, 33-84.

Beversdorf, D. Q., Hughes, J. D., Steinberg, B. A., Lewis, L. D., \& Heilman, K. M. (1999). Noradrenergic modulation of cognitive flexibility in problem solving. NeuroReport, 10, 2763-2767.

Beversdorf, D. Q., White, D. M., Chever, D. C., Hughes, J. D., \& Bornstein, R. A. (2002). Central beta-adrenergic modulation of cognitive flexibility. NeuroReport, 13, 2505-2507.

Birnbaum, S., Gobeske, K. T., Auerbach, J., Taylor, J. R., \& Arnsten, A. F. T. (1999). A role for norepinephrine in stress-induced deficits: $\alpha$-1-Adrenoceptor mediation in the prefrontal cortex. Biological Psychiatry, 46, 1266-1274.

Bowden, E. M., \& Jung-Beeman, M. (2003). Normative data for 144 compound remote associate problems. Behavior Research Methods, Instruments, E Computers, 35, 634-639.

Bunsey, M. D., \& Strupp, B. J. (1995). Specific effects of idazoxan in a distraction task: Evidence that endogenous norepinephrine plays a role in selective attention in rats. Behavioral Neuroscience, 109, 903-911.

Cacioppo, J. T., Malarkey, W. B., Kiecolt-Glaser, J. K., Uchino, B. N., Sgoutas-Emch, S. A., Sheridan, J. F., et al. (1995). Heterogeneity in neuroendocrine and immune responses to brief psychological stressors as a function of autonomic cardiac activation. Psychosomatic Medicine, 57, 154-164.

Cerqueira, J. J., Pêgo, J. M., Taipa, R., Bessa, J. M., Almeida, O. F. X., \& Sousa, N. (2005). Morphological correlates of corticosteroid-induced changes in prefrontal cortex-dependent behaviors. Journal of Neuroscience, 25, 7792-7780.

Clarke, H. F., Dailey, J. W., Crofts, H. S., Robbins, T. W., \& Roberts, A. C. (2004). Cognitive inflexibility after prefrontal serotonin depletion. Science, 304, 878-880.

Corwin, J., \& Bylsma, F. (1993). Translations of excerpts from Andre Rey's psychological examination of traumatic encephalopathy and P.A. Osterrieth's the Complex Figure Test. The Clinical Neuropsychologist, 7, 3-15.

Coull, J. T. (1998). Neural correlates of attention and arousal: Insights from electrophysiology, functional neuroimaging and psychopharmacology. Progress in Neurobiology, 55, 343-361.

Coull, J. T., Buchel, C., Friston, K. J., \& Frith, C. D. (1999). Noradrenergically mediated plasticity in a human attentional neuronal network. Neuroimage, 10, 705-715.

Coull, J. T., Jones, M. E. P., Egan, T. D., Frith, C. D., \& Maze, M. (2004). Attentional effects of noradrenaline vary with arousal 
level: Selective activation of thalamic pulvinar in humans. Neuroimage, 22, 315-322.

Dalley, J. W., Cardinal, R. N., \& Robbins, T. W. (2004). Prefrontal executive and cognitive functions in rodents: Neural and neurochemical substrates. Neuroscience and Biobehavioral Reviews, 28, 771-784.

de Quervain, D. J.-F., Roozendaal, B., Nitsch, R. M., McGaugh, J. L., \& Hock, C. (2000). Acute cortisone administration impairs retrieval of long-term declarative memory in humans. Nature Neuroscience, 3, 313-314.

Dey, M. K. (1978). Interactions of manifest anxiety score with anagram letter transition probability and Thorndike-Lorge frequency of solution word. Journal of General Psychology, 99, 263-270.

Easterbrook, J. A. (1959). The effect of emotion on cue utilization and the organization of behavior. Psychological Review, 66, 183-201.

Elzinga, B. M., \& Roelofs, K. (2005). Cortisol-induced impairments of working memory require acute sympathetic activation. Behavioral Neuroscience, 119, 98-103.

Evers, E. A. T., Cools, R., Clark, L., van der Veen, F. M., Jolles, J., Sahakian, B. J., et al. (2005). Serotonergic modulation of prefrontal cortex during negative feedback in probabilistic reversal learning. Neuropsychopharmacology, 30, $1138-1147$.

Faigel, H. C. (1991). The effect of beta blockade on stress-induced cognitive dysfunction in adolescents. Clinical Pediatrics, 30, 441-445.

Floresco, S. B., Magyar, O., Ghods-Sharifi, S., Vexelman, C., \& Tse, M. T. L. (2006). Multiple dopamine receptor subtypes in the medial prefrontal cortex of the rat regulate set-shifting. Neuropsychopharmacology, 31, 297-309.

Gavurin, E. (1975). Relationship of anagram solving to measures of divergent-production and letter-rearrangement ability. Journal of General Psychology, 92, 231-235.

Goldstein, L. E., Rasmusson, A. M., Bunney, B. S., \& Roth, R. H. (1996). Role of the amygdala in the coordination of behavioral, neuroendocrine, and prefrontal cortical monoamine responses to psychological stress in the rat. Journal of Neuroscience, 16, 4787-4798.

Harleston, B. W., Smith, M. G., \& Arey, D. (1965). Test-anxiety level, heart rate, and anagram problem solving. Journal of Personality and Social Psychology, 36, 551-557.

Hasselmo, M. E., Linster, C., Patil, M., Ma, D., \& Cekic, M. (1997). Noradrenergic suppression of synaptic transmission may influence cortical signal-to-noise ratio. Journal of Neurophysiology, 77, 3326-3339.

Heilman, K. M., Nadeau, S. E., \& Beversdorf, D. Q. (2003). Creative innovation: Possible brain mechanisms. Neurocase, 9, 369-379.

Johnson, A. K., \& Anderson, E. A. (1990). Stress and arousal. In J. T. Cacioppo (Ed.), Principles of psychophysiology: Physical, social and inferential elements (pp. 216-252). New York: Cambridge University Press.

Kelley, B. J., Yeager, K. R., Pepper, T. H., \& Beversdorf, D. Q. (2005). Cognitive impairment in acute cocaine withdrawal. Cognitive and Behavioral Neurology, 18, 108-112.

Kirschbaum, C., Pirke, K. M., \& Hellhammer, D. H. (1993). The "Trier Social Stress Test"-A tool for investigating psychobiological stress responses in a laboratory setting. Neuropsychobiology, 28, 76-81.

Koob, G. F. (1991). Arousal, stress, and inverted U-shaped curves: Implications for cognitive function. In R. G. Lister \& H. J. Weingartner (Eds.), Perspectives on cognitive neuroscience (pp. 301-313). New York: Oxford University Press.
Koob, G. F., Cole, B. J., Swerdlow, N. R., Le Moal, M., \& Britton, K. T. (1990). Stress, performance, and arousal: Focus on CRF. NIDA Research Monograph, 97, 163-176.

Kumar, D., \& Kumari, S. (1988). Problem solving as a function of creativity and personality. Psychological Studies, 33, 157-161.

Lapiz, M. D. S., \& Morilak, D. A. (2006). Noradrenergic modulation of cognitive function in rat medial prefrontal cortex as measured by attentional set shifting capability. Neuroscience, 137, 1039-1049.

Lewis, D. A., \& Morrison, J. H. (1989). Noradrenergic innervation of monkey prefrontal cortex: A dopamine- $\beta$-hydroxylase immunohistochemical study. Journal of Comparative Neurology, 282, 317-330.

Lyons, D. M., Lopez, J. M., Yang, C., \& Schatzberg, A. F. (2000). Stress-level cortisol treatment impairs inhibitory control of behavior in monkeys. Journal of Neuroscience, 20, 7816-7821.

Maheu, F. S., Joober, R., Beaulieu, S., \& Lupien, S. J. (2004). Differential effects of adrenergic and corticosteroid hormonal systems on human short- and long-term declarative memory for emotionally arousing material. Behavioral Neuroscience, 118, 420-428.

Maheu, F. S., Joober, R., \& Lupien, S. J. (2005). Declarative memory after stress in humans: Differential involvement of the beta-adrenergic and corticosteroid systems. Journal of Clinical Endocrinology and Metabolism, 90, 1697-1704.

Martindale, C., \& Greenough, J. (1973). The differential effect of increased arousal on creative and intellectual performance. Journal of Genetic Psychology, 123, 329-335.

Mednick, S. (1962). The associative basis of the creative process. Psychological Review, 69, 220-232.

Murphy, B. L., Arnsten, A. F. T., Jentsch, J. D., \& Roth, R. H. (1996). Dopamine and spatial working memory in rats and monkeys: Pharmacological reversal of stress-induced impairment. Journal of Neuroscience, 16, 7768-7775.

Nieuwenhuis, S., Aston-Jones, G., \& Cohen, J. D. (2005). Decision making, the P3, and the locus coeruleusnorepinephrine system. Psychological Bulletin, 131, 510-532.

Peacock, E. J., \& Wong, P. T. P. (1990). The stress appraisal measure (SAM): A multidimensional approach to cognitive appraisal. Stress Medicine, 6, 227-236.

Ramos, B. P., Colgan, L., Nou, E., Ovadia, S., Wilson, S. R., \& Arnsten, A. F. T. (2005). The beta-1 adrenergic antagonist, betaxolol, improves working memory performance in rats and monkeys. Biological Psychiatry, 58, 894-900.

Reich, J., \& Zautra, A. (2002). Arousal and the relationship between positive and negative affect: An analysis of the data of Ito, Cacioppo, and Lang (1998). Motivation and Emotion, 26, 209-222.

Rey, A. (1941). L'examen psycholgique dans les cas d'encephalopathie tramatique. Archives de Psychologie, 28, 286-340.

Roozendaal, B., McReynolds, J. R., \& McGaugh. J. L. (2004). The basolateral amygdala interacts with the medial prefrontal cortex in regulating glucocorticoid effects on working memory impairment. Journal of Neuroscience, 24, 1385-1392.

Segal, M., \& Bloom, F. E. (1976). The action of norepinephrine in the rat hippocampus. IV. The effects of locus coeruleus stimulation on evoked hippocampal unit activity. Brain Research, 107, 513-525.

Shaw, G., \& Conway, M. (1990). Individual differences in nonconscious processing: The role of creativity. Personality and Individual Differences, 11, 407-408. 
Silver, J. A., Hughes, J. D., Bornstein, R. A., \& Beversdorf, D. Q. (2004). Effect of anxiolytics on cognitive flexibility in problem solving. Cognitive and Behavioral Neurology, 17, 93-97.

Skosnik, P. D., Chatterton, R. T., Jr., Swisher, T., \& Park, S. (2000). Modulation of attentional inhibition by norepinephrine and cortisol after psychological stress. International Journal of Psychophysiology, 36, 59-68.

Smith, A., \& Nutt, D. (1996). Noradrenaline and attention lapses. Nature, 380, 291.
Stemme, A., Deco, G., Busch, A., \& Schneider, W. X. (2005). Neurons and the synaptic basis of the fMRI signal associated with cognitive flexibility. Neuroimage, 26, 454-470.

Tomasini, J. (1973). Effect of peer-induced anxiety on a problem-solving task. Psychological Reports, 33, 355-358.

Young, A. H., Sahakian, B. J., Robbins, T. W., \& Cowen, P. J. (1999). The effects of chronic administration of hydrocortisone on cognitive function in normal male volunteers. Psychopharmacology, 145, 260-266. 